\title{
Painful myositis in the anti-synthetase syndrome with anti-PL12 antibodies
}

\author{
P Mehta ${ }^{1}$, L Patel ${ }^{1}$, F Roncaroli ${ }^{2}, M C$ Pickering $^{3}$, A Brand $^{1 *}$ \\ From 5th European Workshop on Immune-Mediated Inflammatory Diseases \\ Sitges-Barcelona, Spain. 1-3 December 2010
}

The idiopathic inflammatory myopathies (IIMs) are characterized by muscle weakness, electromyography (EMG), histological and biochemical features of muscle inflammation. Their classification is evolving due to the myositis-associated autoantibody panel. Anti-aminoacyl-tRNA synthetase (ARS) antibodies are detected in $25-30 \%$ patients and define the anti-synthetase syndrome (ASS), a spectrum of interstitial lung disease (ILD), arthritis, fever, Raynaud's phenomenon and mechanic's hands. Anti-Jo1 antibodies are most common (20\%), whereas anti-PL12 antibodies (alanyl-tRNA synthetase) occur in $<3 \%$ cases.

We report a 58-year-old male with IIM associated with anti-PL12 antibodies, who presented with a threeweek history of severe widespread muscle pain, without overt muscle weakness. He also reported polyarthralgia, exertional dyspnoea, dry cough and weight loss. Examination revealed low-grade pyrexia, bi-basal crepitations and exquisitely tender muscles. Notably, there was no proximal muscle weakness or neurological deficit.

Investigations revealed marked acute phase response (CRP $121 \mathrm{mg} / \mathrm{l}$, ESR $79 \mathrm{~mm} / \mathrm{hr}$ ), polyclonal hypergammaglobulinaemia and raised ALT 276 U/l (0-40). Serology demonstrated high-titre anti-nuclear antibodies (1:1280, speckled). Serum Western blotting revealed anti-PL12 antibodies. No other autoantibodies were detected. Creatinine kinase was elevated 936 U/l (0-200), EMG was consistent with myositis. MRI thighs demonstrated high signal within the semitendinosis muscle.

Muscle histology confirmed a florid inflammatory myopathy with disruption, oedema and considerable macrophage and lymphocytic infiltrate of perimysial connective tissue - typical features of ASS. Spirometry was restrictive with reduced corrected gas transfer. CT chest showed non-specific interstitial pneumonia.

${ }^{1}$ Rheumatology, Chelsea and Westminster Hospital, London, UK

Full list of author information is available at the end of the article
Prednisolone 60mg daily, resulted in improvement of dyspnoea and normalisation of CRP and CK.

Significant muscle pain (requiring morphine) was steroid-responsive, but only to high doses, with recurrence on dose reduction. Pulsed cyclophosphamide was commenced, with improvement in muscle pain after the first dose.

Severe muscular pain and tenderness in the absence of weakness, is atypical of ASS and has not been previously reported. Anti-PL12 antibodies are rare, therefore their associated clinical features are not well-described, unlike the Jo-1 syndrome. ILD is common, usually precedes muscle involvement and may occur in isolation. Previous reports suggest that muscle involvement is uncommon and mild (often subclinical). Further reports and prospective studies will help refine the clinical phenotype of antiPL12 antibody-positive ASS subtype.

\section{Author details}

${ }^{1}$ Rheumatology, Chelsea and Westminster Hospital, London, UK.

${ }^{2}$ Histopathology, Imperial College London, UK. ${ }^{3}$ Rheumatology, Imperial College London, UK.

Published: 25 November 2010

doi:10.1186/1479-5876-8-S1-P52

Cite this article as: Mehta et al:: Painful myositis in the anti-synthetase syndrome with anti-PL12 antibodies. Journal of Translational Medicine 2010 8(Suppl 1):P52. 